Pacific Journal of Mathematics

INNER FUNCTIONS UNDER UNIFORM TOPOLOGY 


\title{
INNER FUNCTIONS UNDER UNIFORM TOPOLOGY
}

\author{
Domingo A. Herrero
}

\begin{abstract}
The structure of the space $\mathscr{F}$ of all inner functions in the unit disc $D=\{z:|z|<1\}$ under the metric topology induced by the $H^{\infty}$-norm is considered. It is proven that if two inner functions $p$ and $q$ belong to the same component of $\mathscr{F}$, then the variation of $p / q$ on each open arc of $\partial D$ (the boundary of $D$ in the complex plane $C$ ) where they can be continued analytically is bounded by a constant $C=C(p, q)$, independent of the arc. This criterion is used to show that a component of $F$ can contain nothing but Blaschke products with infinitely many zeroes, exactly one (up to a constant factor) singular inner function or infinitely many pairwise coprime singular inner functions.
\end{abstract}

The reader is assumed to be familiar with the basic theory of the space $H^{\infty}$. We recall that the canonical form of an inner function is: $q=\lambda b r=\lambda b d s$, where $\lambda \in \partial D$ and

$$
b(z)=\prod_{k} b_{k}(z)=\prod_{k} b\left(z, a_{k}\right)=\prod_{k} \overline{a_{k}} /\left|a_{k}\right| \cdot\left(a_{k}-z\right) /\left(1-\overline{a_{k}} z\right),
$$

$\left(\overline{a_{k}} /\left|a_{k}\right|=-1\right.$, if $\left.a_{k}=0\right), a_{k} \in D$ and $\sum_{k}\left(1-\left|a_{k}\right|\right)<\infty ; b(z)$ is the Blaschke product of $q$ and the $b_{k}$ 's are the Blaschke factors;

$$
r(z)=e(z, \mu)=\exp \left\{\int_{0}^{2 \pi}\left(z+e^{i \theta}\right) /\left(z-e^{i \theta}\right) d \mu(\theta)\right\},
$$

where $\mu$ is a nonnegative singular Borel measure on $\partial D ; r(z)$ is the singular part of $q$. If $\mu=\nu+\nu_{1}$, where $\nu$ and $\nu_{1}$ are the continuous and the purely atomic part, resp., of $\mu$, then

(3) $s(z)=e(z, \nu)$ is the continuous singular part of $q$, and

(4) $d(z)=e\left(z, \nu_{1}\right)=\Pi_{j} d_{j}(z)=\Pi_{j} d\left(z ; \theta_{\jmath}, l_{j}\right)$, where $d(z ; \theta, l)=$ $\exp \left\{l\left(z+e^{i \theta}\right) /\left(z-e^{i \theta}\right)\right\}, 0 \leqq \theta<2 \pi$ and $l>0 ; d(z)$ is the atomic singular part of $q$. Clearly, $\sum_{j} l_{j}=\left\|\nu_{1}\right\|<\infty$; it will be assumed that $\theta_{j} \neq \theta_{h}$, whenever $j \neq h$.

The subsets of $\mathscr{F}$ containing all those functions defined by the conditions (1), (2), (3), and (4) (or the constant multiples of such functions) will be denoted by $\mathscr{F}_{B}, \mathscr{F}_{S}, \mathscr{F}_{C}$, and $\mathscr{F}_{A}$, resp.

It is well-known that the inner function $q(z)$ can be continued analytically across (a neighborhood of) the point $\lambda \in \partial D$ if and only if $\lambda \notin \operatorname{Sp}(q)=\operatorname{supp}(\mu) \cup$ closure $\left\{a_{k}\right\}$; furthermore, if $\operatorname{Sp}(q) \cap \partial D \neq$ $\operatorname{Sp}(p) \cap \hat{o} D(p, q \in \mathscr{F})$, then $\|q-p\|_{\infty}=2$, and $p$ and $q$ belong to different components of $\mathscr{F}$.

Let $\mathscr{F}_{\Gamma}(\Gamma$ a closed subset of $\partial D)$ be the set of all inner functions such that $\operatorname{Sp}(q) \cap \partial D=\Gamma$. Clearly, $\mathscr{F}_{\phi}$ is the family of all Blaschke 
products of finite order, and it is not difficult to see that $\mathscr{F}_{\phi}=$ $\bigcup_{n=0}^{\infty} \mathscr{F}_{n}$ (disjoint union!), where $\mathscr{F}_{n}$ is the family of Blaschke products of order $n$ and (for each $n$ ) $\mathscr{F}_{n}$ is an acrwise connected open and closed component of $\mathscr{F}$, topologically homeomorphic to $\partial D \times D^{n}$ (see $[2 ; 4]$ ).

During the "Sesquicentennial Seminar in Operator Theory" (Indiana University, Bloomington, Indiana, 1969-1970), R. G. Douglas asked for a characterization of the components of $\mathscr{F} / \mathscr{F}_{\phi}$ containing a singular inner function; in particular, he asked whether or not every component of $\mathscr{F} / \mathscr{F}_{\phi}$ contains a singular inner function. Here we partially answer the first question. A first counterexample to the second question was given by $\mathrm{D}$. Sarason; his unpublished result follows from his paper [5] (some components of $\mathscr{F} / \mathscr{F}_{\phi}$ contain nothing but Blaschke products whose zeroes converge nontangentially to $z=1$ ). We shall give another counterexample by using a different approach.

We want to express our gratitude to Professors R. G. Douglas, D. Sarason, and A. E. Tong for several helpful discussions; especially to D. Sarason who made available his unpublished paper [5]. We are also grateful to the referee who made several suggestions.

1. Components of $\mathscr{F}$ containing exactly one, or $\underline{c}(\underline{c}=$ the power of the continuum) singular inner functions. We shall need a remarkable classical result due to O. Frostman.

THEOREM $1.1([3, \S 59$, p. 111]). Let $q \in \mathscr{F}$ and let

$$
q_{a}(z)=[q(z)-a] /[1-\bar{a} q(z)], \quad a \in D .
$$

Then, for all $a \in D$, except for a subset of logarithmic capacity zero, $q_{a} \in \mathscr{F}_{B}$.

For a precise definition of logarithmic capacity of a subset $\Lambda$ of the complex plane, see [3;6]. For our purposes, it is enough to recall that, if $\log$ cap $\Lambda=0$, then $\Lambda$ is a "very small" subset of $\boldsymbol{C}$; in fact, $\Lambda$ has planar Lebesgue measure zero and, moreover, the projection of $A$ on any line of $C$ has linear measure zero. Thus, Theorem 1.1 implies, in particular, that $\mathscr{F}_{B}$ is dense in $\mathscr{F}$.

We are going to analyze the behavior of an inner function $q$ on a closed arc $\Gamma=\left[e^{i \alpha}, e^{i \beta}\right](0 \leqq \alpha<2 \pi, \alpha<\beta<\alpha+2 \pi)$, contained in $\partial D \backslash \operatorname{Sp}(q)$. For an arbitrary continuously differentiable unimodular function $u\left(e^{i \theta}\right)$ defined in a neighborhood (in $\partial D$ ) of $\Gamma$, we define the variation of $u$ on $\Gamma$ as

$$
M(u ; \alpha, \beta)=\arg u\left(e^{i \beta}\right)-\arg u\left(e^{i \alpha}\right)=\int_{\alpha}^{\beta} d \arg u\left(e^{i \theta}\right),
$$


where $\arg u\left(e^{i \theta}\right)$ denotes any continuously differentiable determination of the argument of $u\left(e^{i \theta}\right)$.

LEMma 1.2. Let $p$ and $q$ be two inner functions and let $\Gamma$ be as above. Then

(i) $M(q ; \alpha, \beta) \geqq 0$;

(ii) If $\Gamma \subset \partial D \backslash \operatorname{Sp}(p), \quad M(p q ; \alpha, \beta)=M(p ; \alpha, \beta)+M(q ; \alpha, \beta) \geqq$ $M(q ; \alpha, \beta)$;

(iii) If $p$ belongs to the component of $q$, then $\operatorname{Sp}(p) \cap \partial D=$ $\operatorname{Sp}(q) \cap \partial D$ and there exists a constant $C=C(p, q)$ such that $|M(q ; \alpha, \beta)-M(p ; \alpha, \beta)|=|M(q / p ; \alpha, \beta)| \leqq C$, and $C$ is independent of $\Gamma$.

Proof. (i ) This is trivial for finite Blaschke products. If $q(z)=$ $\Pi_{k=1}^{\infty} b\left(z, a_{k}\right)$ is an infinite Blaschke product such that $\Gamma \subset \partial D \backslash \operatorname{Sp}(q)$, then observe that $\left\{\prod_{k=1}^{n} b\left(z, a_{k}\right)\right\}_{n=1}^{\infty}$ converges to $q(z)$ uniformly on $\Gamma$, from which the result follows. Finally, if $q$ is any inner function, then we can find (by Frostman's theorem) a sequence $\left\{a_{n}\right\} \subset D$ converging to zero, such that $q_{n}=q_{a_{n}}$ (defined by (1)) is a Blaschke product, for all $n$. Clearly, $q_{n}$ belongs to the component of $q$ and therefore (see the introduction) $\operatorname{Sp}\left(q_{n}\right) \cap \partial D=\operatorname{Sp}(q) \cap \partial D$. Since the result is true for all $q_{n}, n=1,2, \cdots$ and $\left|q_{n}(z)-q(z)\right| \rightarrow 0$, as $n \rightarrow \infty$, uniformly on $\Gamma$, the result is also true for $q$.

(ii) This follows immediately from (i).

(iii) Let $\mathscr{C}_{0}(q)=\left\{q^{\prime} \in \mathscr{F}:\left\|q^{\prime}-q\right\|_{\infty}<1\right\}$ and, by induction, define $\mathscr{C}_{n}(q)=\left\{q^{\prime \prime} \in \mathscr{F}:\left\|q^{\prime \prime}-q^{\prime}\right\|_{\infty}<1\right.$, for some $\left.q^{\prime} \in \mathscr{C}_{n-1}(q)\right\}, n=1,2, \cdots$, and $\mathscr{C}(q)=\bigcup_{n=0}^{\infty} \mathscr{C}_{n}(q)$. Clearly, $\mathscr{C}(q)$ is open and closed in $\mathscr{F}$ and contains the component of $q$. Moreover, $\operatorname{Sp}\left(q^{\prime}\right) \cap \partial D=\operatorname{Sp}(q) \cap \partial D$, for all $q^{\prime} \in \mathscr{C}(q)$.

Let $q^{\prime} \in \mathscr{C}_{0}(q)$; then for every $e^{i \theta} \in \Gamma,\left|q\left(e^{i \theta}\right)-q^{\prime}\left(e^{i \theta}\right)\right| \leqq\left\|q-q^{\prime}\right\|_{\infty}<1$, and therefore, for a suitable continuously differentiable definition of the argument, we have $\left.\mid \arg q\left(e^{i \theta}\right) / q^{\prime}\left(e^{i \theta}\right)\right\}<\pi / 3$. Hence, $\left|M\left(q / q^{\prime} ; \alpha, \beta\right)\right|=$ $\left|\arg q\left(e^{i \beta}\right) / q^{\prime}\left(e^{i \beta}\right)-\arg q\left(e^{i \alpha}\right) / q^{\prime}\left(e^{i \alpha}\right)\right| \leqq\left|\arg q\left(e^{i \beta}\right) / q^{\prime}\left(e^{i \beta}\right)\right|+\left|\arg q\left(e^{i \alpha}\right) / q^{\prime}\left(e^{i \alpha}\right)\right|<$ $2 \pi / 3$.

By an elementary inductive argument, it follows that $\left|M\left(q / q^{\prime \prime} ; \alpha, \beta\right)\right|<$ $2 \pi(n+1) / 3$, for all $q^{\prime \prime} \in \mathscr{C}_{n}(q), n=0,1,2, \cdots$, whence the result follows.

THeOREM 1.3. Let $d(z)=d(z ; 0,1)$. Then, for each positive $r$, the component of $d$ is isometric to the component of $d^{r}$; however, if $r \neq 1, d$ and $d^{r}$ belong to different components of $\mathscr{F}$. Thus, if $0<$ $r<1$, then the subset $\left\{q d^{r} \in(\right.$ component of $\left.d)\right\}$ is isometric to the whole component of $d \cdot d^{r}$ is the only (up to a constant factor) singular inner function in its own component, for each $r>0$. 
Proof. If $b(z)$ is a Blaschke factor, then the mapping $q(z) \rightarrow q(b(z))$ is an isometry from $\mathscr{F}$ onto itself. If the zero of $b(z)$ is the point $a=(1-r) /(1+r) \in D(r>0)$, then $d(b(z))=d^{r}(z)$, which proves the first part.

Observe that $\operatorname{Sp}(d)=\{1\}$ and $M\left(d^{t} ; 1 / n, \pi\right) \rightarrow+\infty$, as $n \rightarrow \infty$, for each positive $t$; hence, $d^{r}$ and $d^{r+t}$ cannot belong to the same component, as follows from Lemma 1.2. Since $\mathscr{F}_{S} \cap \mathscr{F}_{11\}}=\left\{\lambda d^{t}: \lambda \in\right.$ $\partial D, t>0\}$, we conclude that every singular inner function in the component of $d^{r}$ is a constant multiple of $d^{r}$.

The remaining statements are clear now.

COROLlaRY 1.4. Let $q \in \mathscr{F}_{S}$ and assume that $\left\{\theta: e^{i \theta} \in \operatorname{Sp}(q)\right\}$ is well-ordered (with the usual order of the interval $[0,2 \pi)$ ). Then the only singular inner functions in the component of $q$ are the constant multiples of $q$.

Proof. Clearly, $\operatorname{Sp}(q)$ is countable and therefore $q$ has the form $q(z)=\lambda \Pi_{j} d\left(z ; \theta_{j}, l_{j}\right)=\lambda \Pi_{j} d_{j}(z)$ (i.e., $\left.q \in \mathscr{F}_{A}\right)$. Moreover, if $p \in \mathscr{F}_{S}$ belongs to the component of $q$, then $p(z)=\lambda^{\prime} \Pi_{h} d\left(z ; \theta_{h}^{\prime}, l_{j}^{\prime}\right)$, where $\lambda^{\prime} \in \partial D$ and $\operatorname{clos}\left\{e^{i \theta_{h}^{\prime}}\right\}=\operatorname{clos}\left\{e^{i \theta} j\right\}=\operatorname{Sp}(q)$.

Let $q(z)=\lambda q_{1}(z) q_{1}^{\prime}(z)$, where $q_{1}(z)$ is the product of all $d_{j}$ 's corresponding to the isolated points of $\operatorname{Sp}(q)$. It is not difficult to see, by using Lemma 1.2 and the arguments of the proof of Theorem 1.3, that $p(z)=\lambda^{\prime} q_{1}(z) p_{1}^{\prime}(z)$, where $\operatorname{Sp}\left(p_{1}^{\prime}\right)=\operatorname{Sp}\left(q_{1}^{\prime}\right) \subset \operatorname{Sp}(q)^{\prime}$ (here $\Gamma^{\prime}$ denotes the derived set of the set $\Gamma$ ).

Now, let $q(z)=\lambda q_{1}(z) q_{2}(z) q_{2}^{\prime}(z)$, where $q_{2}(z)$ is the product of the $d_{j}^{\prime}$ 's corresponding to the isolated points of $\operatorname{Sp}\left(q_{1}^{\prime}\right)$. Using the above arguments and the fact that, if $e^{i \theta} \in \operatorname{Sp}\left(q_{1}^{\prime}\right)$, then the arc $\left(e^{i \theta}, e^{i(\theta+\varepsilon)}\right]$ (for some $\varepsilon=\varepsilon(\theta)>0$ ) does not intersect $\operatorname{Sp}(q)$ (here we are using the "well-order property"!), we see that $p(z)=\lambda^{\prime} q_{1}(z) q_{2}(z) p_{2}^{\prime}(z)$.

The result follows by a transfinite inductive argument.

It is clear that, if $q \in \mathscr{F}$ is nonconstant, then $q(D)$ is an open subset of $D$; in general, $q(D) \neq D$ (namely, if $q$ is singular, then $0 \notin q(D)$ ). In [6, Theorem 10], W. Seidel proved that if $q$ is a nonconstant inner function and $D \backslash q(D)$ contains more than one point, then $\operatorname{Sp}(q) \cap \partial D$ is a nonempty perfect subset of $\partial D$. The set $D \backslash q(D)$ has been completely determined by 0 . Frostman $([3, \S 61$, p. 113]; see also [6, Theorem 13]): If $q \in \mathscr{F}$ is nonconstant, then $D \backslash q(D)$ is a closed subset of $D$ of logarithmic capacity zero; conversely, if $\Lambda$ is a closed subset of $D$ and $\log$ cap $\Lambda=0$, then the uniformizer of the Riemann surface $D \backslash \Lambda$ is an inner function $q$ such that $D \backslash q(D)=\Lambda$. Moreover, if $\Lambda$ is compact, then $\operatorname{Sp}(q) \cap \partial D$ has linear measure zero (see [6, p. 218]). The uniformizer of $D \backslash\{a\}(a \in D)$ can be taken equal 
to $d_{(-a)}(z ; \theta, l)=q(z)$ (defined by (1); $\theta$ and $l>0$ can be arbitrarily chosen); in this case $\operatorname{Sp}(q)=\left\{e^{i \theta}\right\}$ consist of a single point and we know (by Theorem 1.3) that the component of $q$ contains exactly one (up to a constant factor) singular inner function. On the other hand, if $\Lambda$ is a compact subset of logarithmic capacity zero of $D$, and $A$ contains more than one point, the uniformizer of $D \backslash \Lambda, p(z)$ is an inner function such that $\operatorname{Sp}(p)$ is a nonempty perfect subset of $\partial D$ of linear measure zero; since, for every $a \in \Lambda, p_{a} \in \mathscr{F}_{S}$, we conclude that the component of $p$ contains (at least!) two coprime singular inner functions. In fact, if $q=r s$ and $q_{a}=r t$, where $q, r, s, t \in \mathscr{F}$ and $a \neq 0$, then $q-a=r s-a=(1-\bar{a} q) r t$; hence $t(1-\bar{a} q)=s-a / r \in H^{\infty}$. It follows that $r$ and $a / r$ belong to $H^{\infty}$, but this is impossible unless $r$ is a constant. In other words, $q$ and $q_{a}$ ard coprime (this example and Theorem 1.5 below are due to D. Sarason).

Corollary 1.4 may be considered an improvement of the above mentioned result of $W$. Seidel for a very particular class of singular inner functions (in fact, Seidel's result can be reformulated as: If $\operatorname{Sp}(q) \cap \partial D$ is not perfect, then $D \backslash q(D)$ contains, at most, one point) and we guess that the "well-order" condition could be replaced by the weaker condition "Sp $(q) \cap \partial D$ is countable"; however, Lemma 1.2 is not sufficient to prove this stronger conjecture. On the other hand, an analysis of the function $p_{a}$ of the above example shows that no "reasonable" condition weaker than "countable" can work to get the same result.

Let $p(z)$ be the uniformizer of $D \backslash \Lambda$, where $\Lambda$ is any nonempty perfect subset of $D$ of logarithmic capacity zero (e.g., take as $\Lambda$ a suitable "Cantor type" subset of the real interval $[0,1 / 2]$; see [3;6]). Then, for each $a \in \Lambda, p_{a}$ is a singular inner function. Since $c(\Lambda)=\underline{c}$, the power of the continuum, it follows from the previous observations that

THEOREM 1.5 (D. Sarason). There exists a compact of $\mathscr{F}$ containing $\underline{c}$ pairwise coprime singular inner functions.

The perfect set $\Lambda$ can be replaced by a finite subset or by a sequence of points in $D$ converging to $\partial D$. This suggests that, for each $n=0,1,2, \cdots, \boldsymbol{\aleph}_{0}$, there exists a component of $\mathscr{F}$ containing exactly $n$ coprime singular inner functions or, at least, exactly $n$ "essentially different" (i.e., $p / q$ is not a constant) singular inner functions, but we have been unable to prove it.

From Theorem 1.1, we obtain 
CoROLlary 1.6.

(i) $\mathscr{F}_{s}$ is a closed nowhere dense subset of $\mathscr{F}$.

(ii) $\mathscr{F}_{B}$ is a dense, but not open subset of $\mathscr{F}$.

Proof. By the observations following Theorem 1.1, we only have to prove that $\mathscr{F}_{S}$ is closed and $\mathscr{F}_{B}$ is not open. The first fact is immediate, because $\mathscr{F}_{S}$ is clearly closed with respect to the compactopen topology restricted to $\mathscr{F}$ and the norm-topology in $H^{\infty}$ is stronger than the compact-open topology.

To see that $\mathscr{F}_{B}$ is not open, write $d(z ; 0,1)=\prod_{j=1}^{\infty} d_{j}(z)$, where $d_{j}(z)$ is the $2^{j}$-root of $d(z ; 0,1)$, and set $b(z)=\prod_{j=1}^{\infty} p_{j}(z), p_{j}(z)=\left[d_{j}(z)-\right.$ $1 / 2 j] /\left[1-(1 / 2 j) d_{j}(z)\right]$. It follows from Theorem 1.3 that $p_{j} \in \mathscr{F}_{B}$, for all $j$, and $b=\prod_{j} p_{j} \in \mathscr{F}_{B}$. Given any $\varepsilon>0$, choose $n$ so that $2 / n<\varepsilon$. Then $\left[\prod_{j \neq n} p_{j}\right] d_{n} \notin \mathscr{F}_{B}$ and $\left\|b-\left[\prod_{j \neq n} p_{j}\right] d_{n}\right\|_{\infty}=\left\|\left(d_{n}\right)_{(1 / 2 n)}-d_{n}\right\|_{\infty}<$ $2 / n<\varepsilon$.

Therefore, $b(z)$ does not belong to the interior of $\mathscr{F}_{B}$.

We close this section with two conjectures:

(1) The component, in $\mathscr{F}_{s}$, of a singular inner function $p$ is the set of the constant multiples of $p$ (i.e., $\mathscr{F}_{S}$ is "essentially" a totally disconnected space).

(2) $\mathscr{F}_{C}$ and $\mathscr{F}_{A}$ are closed in $\mathscr{F}$.

\section{Components contained in $\mathscr{F}_{B}$.}

THEOREM 2.1. Given any sequence $0 \leqq r_{1} \leqq r_{2} \leqq \cdots \leqq r_{k} \leqq \cdots<1$ of radii such that $\sum_{k=1}^{\infty}\left(1-r_{k}\right)<\infty$, it is possible to choose $\underline{c}$ sequences $\left\{\theta_{k}(t): 0 \leqq t<\infty\right\}$ of arguments such that, for each $t \in[0, \infty)$, the component of

$$
b_{t}(z)=\prod_{k=1}^{\infty} b\left(z, r_{k} \exp \left\{i \theta_{k}(t)\right\}\right)
$$

in $\mathscr{F}$ is contained in $\mathscr{F}_{B}$. Moreover, if $t \neq t^{\prime}$, then $b_{t}$ and $b_{t}$, belong to different components.

Proof. First of all observe that $\prod_{k=1}^{\infty} b\left(z, r_{k}\right)$ converges uniformly on each of the subsets $A_{n}=\left\{z:|z| \leqq 1,|1-z| \geqq 2^{-n}\right\}$. Therefore, we can choose $k_{0}=0<k_{1}<k_{2}<\cdots$ in such a way that

$$
\begin{aligned}
&\left|M\left(\prod_{k=m_{1}}^{m_{2}} b\left(e^{i \theta}, r_{k}\right) ; 2^{-n}, 2 \pi-2^{-n}\right)\right|<2^{-n}, n=1,2, \cdots, \quad \text { and } \\
& 2 \pi\left(m_{2}-m_{1}+1\right)-2^{-n}<\left|M\left(\prod_{k=m_{1}}^{m_{2}} b\left(e^{i \theta}, r_{k}\right) ;-2^{-n}, 2^{-n}\right)\right| \\
&<2 \pi\left(m_{2}-m_{1}+1\right), n=1,2, \cdots,
\end{aligned}
$$

whenever $k_{n} \leqq m_{1} \leqq m_{2}<\infty$. 
Define $b(z)=b_{0}(z)=\prod_{h=1}^{\infty} b_{h}^{\prime}(z)$, where

$$
b_{h}^{\prime}(z)=\prod\left\{b\left(z, r_{k} \exp \left[i 2^{-h}\right]\right): k_{h-1}<k \leqq k_{h}\right\}, h=1,2 \cdots .
$$

Then the first inequality of (2) implies that $M\left(b_{0}\left(e^{i \theta}\right) ; \pi, 2 \pi-\varepsilon\right)$ is uniformly bounded with respect to $\varepsilon, 0<\varepsilon<\pi$. Since $\operatorname{Sp}\left(b_{0}\right) \cap \partial D=\{1\}$, it is easy to see from Lemma 1.2 and the properties of the functions $d(z ; 0, l), l>0$, that the component of $b_{0}$ in $\mathscr{F}$ only contains Blaschke products. It is also clear that this property of $b_{0}$ only depends on the fact that the sequence $\left\{k_{h}\right\}_{h=0}^{\infty}$ tends to infinity rapidly enough. Thus, if $k_{h}(t)$ denotes the integer part of $k_{h} h^{t}, 0 \leqq t<\infty$, and $b_{t}$ is defined by $b_{t}(z)=\prod_{h=1}^{\infty} b_{t, h}^{\prime}(z)$, where $b_{t, h}^{\prime}(z)=\Pi\left\{b\left(z, r_{k} \exp \left[i 2^{-h}\right]\right): k_{h-1}(t)<\right.$ $\left.k \leqq k_{h}(t)\right\}$, then the component of $b_{t}$ is also contained in $\mathscr{F}_{B}$ and $\operatorname{Sp}\left(b_{t}\right) \cap \partial D=\{1\}$.

Finally observe that, if $0 \leqq t<t^{\prime}<\infty$, then the second inequality of (2) implies that $M\left(b_{t} / b_{t^{\prime}} ; 2^{-n}, \pi\right) \cong 2 \pi\left\{k_{n-1}(t)-k_{n-1}\left(t^{\prime}\right)+(1 / 2)\left[k_{n}(t)-\right.\right.$ $\left.\left.k_{n-1}(t)\right]-(1 / 2)\left[k_{n}\left(t^{\prime}\right)-k_{n-1}\left(t^{\prime}\right)\right]\right\}=\pi\left\{k_{n-1}(t)-k_{n-1}\left(t^{\prime}\right)+k_{n}(t)-k_{n}\left(t^{\prime}\right)\right\}=$ $-\pi\left\{k_{n}\left(n^{t^{\prime}}-n^{t}\right)+k_{n-1}\left((n-1)^{t^{\prime}}-(n-1)^{t}\right)+F\left(n ; t, t^{\prime}\right)\right\}$, where $F\left(n ; t, t^{\prime}\right)=$ $0\left(k_{n}\right)$; hence, $M\left(b_{t} / b_{t} ; 2^{-n}, \pi\right)$ tends to $-\infty$, as $n$ tends to $\infty$. It follows from Lemma 1.2 that $b_{t}$ cannot belong to the component of $b_{t^{\prime}}$.

THEOREM 2.2. If $b(z)=\prod_{k=1}^{\infty} b\left(z, a_{k}\right)$ has the properties:

(1) The component of $b$ in $\mathscr{F}$ is contained in $\mathscr{F}_{B}$,

(2) $a_{k} \neq 0$ for all $k$ and $\pi>\arg a_{1}>\arg a_{2}>\cdots>\arg a_{k}>$ $\arg a_{k+1}>\cdots>0$, and $\lim \arg a_{k}=0$, and

(3) $M(b ; \pi, 2 \pi-\varepsilon)$ is uniformly bounded with respect to $\varepsilon, 0<$ $\varepsilon<\pi$; then there exists $\underline{c}$ subproducts $b_{\omega}(0 \leqq \omega \leqq \pi / 4)$ of $b$ enjoying the properties (1), (2), and (3); moreover, if $\omega \neq \omega^{\prime}$, then $b_{\omega}$ and $b_{\omega^{\prime}}$ belong to different component of $\mathscr{F}$.

It is completely apparent that, if $b(z)$ is a Blaschke product satisfying any of the properties (1), (2), or (3), then the same result is true for every subproduct of $b$ with infinitely many zeroes. Therefore, we only have to show that the subproducts of $b$ can be chosen in such a way that they belong to different components. The proof of the next lemma is a minor modification of the proof given in [1].

Lemma 2.3. Let $N$ be the set of natural numbers. There exist c subsets $\left\{A_{\omega}: 0 \leqq \omega \leqq \pi / 4\right\}$ of $N$ such that, if $\omega \neq \omega^{\prime}$, then

(i) $A_{\omega} \cap A_{\omega^{\prime}}$ is finite and

(ii) given any $N \in N, A_{\omega}$ contains a finite sequence of $N$ consecutive numbers $n_{N}, n_{N}+1, \cdots, n_{N}+N-1$ which are not in $A_{\omega^{\prime}}$. 
Proof. Enumerate the points with integral coordinates in $\{(x, y): 0 \leqq x \leqq y\}$ as follows:

$$
\begin{aligned}
& 1 \rightarrow(0,0) \\
& 2 \rightarrow(0,1), \quad 3 \rightarrow(1,1) \\
& 4 \rightarrow(0,2), \quad 5 \rightarrow(1,2), \quad 6 \rightarrow(2,2) \\
& 7 \rightarrow(0,3), \quad 8 \rightarrow(1,3), \quad 9 \rightarrow(2,3), \quad 10 \rightarrow(3,3) . \text { etc. }
\end{aligned}
$$

Let $P_{0}=\left\{(x, y): y>x^{2}\right\}$ and let $P_{\omega}, 0 \leqq \omega \leqq \pi / 4$, be the result of rotating $P_{0}$ in $-\omega$ about the origin. Now it is enough to take $A_{\omega}$ equal to the subset of numbers in the above lattice lying inside $P_{\omega}$.

Proof of Theorem 2.2. Properties (2) and (3) show that $b(z)$ can be factored as $b(z)=b^{\prime}(z) \cdot b^{\prime \prime}(z)$, where $b^{\prime}(z)$ is a finite or infinite subproduct and $b^{\prime \prime}(z)=\prod_{k=1}^{\infty} b\left(z, a_{k}^{\prime \prime}\right)$ satisfies (1), (2) (with $a_{k}$ replaced by $\left.a_{k}^{\prime \prime}\right)$, and (3), and moreover,

$$
\left|M\left(b^{\prime \prime} ; \arg a_{k}^{\prime \prime}, \pi\right)-2(k-1 / 2) \pi\right|<2 \pi \text {. }
$$

Set $b_{\omega}(z)=b^{\prime}(z) \cdot \Pi\left\{b\left(z, a_{k}^{\prime \prime}\right): k \in A_{\omega}\right\}, 0 \leqq \omega \leqq \pi / 4$, where $A_{\omega}$ is the subset of $N$ defined in Lemma 2.3. Now observe that the definition of $A_{\omega}$ and (3) imply that, if $0 \leqq \omega<\omega^{\prime} \leqq \pi / 4$, then

$$
\lim \sup \left\{M\left(b_{\omega} / b_{\omega^{\prime}} ; \arg \alpha_{k}^{\prime \prime}, \pi\right): k \rightarrow \infty\right\}=+\infty
$$

or $\lim \inf \left\{M\left(b_{\omega} / b_{\omega^{\prime}} ; \arg a_{k}^{\prime \prime}, \pi\right): k \rightarrow \infty\right\}=-\infty$. Thus, the result follows from Lemma 1.2.

REMARK. It is completely apparent that the same argument can be used to prove, e.g., that $\mathscr{F}$ has $\underline{c}$ components containing nothing but Blaschke products whose sequences of zeroes converges nontangentially to $z=1$. To see this, set $b(z)=\prod_{k=1}^{\infty} b\left(z, r_{k}\right)$, where $\left\{r_{k}\right\}_{k=1}^{\infty}$ is a sequence of radii converging "very rapidly" to 1 so that, for a suitable sequence of arguments $\left\{\theta_{n}\right\}_{n=1}^{\infty}$, decreasing to zero, $M\left(d\left(e^{i \theta} ; 0,1 / n\right) / b\left(e^{i \theta}\right) ; \theta_{n}, \pi\right\}>n, n=1,2, \cdots$. Clearly, the same result is true for any of the subproducts $b_{\omega}$ of $b$ (defined as in the proof of Theorem 2.2), and the result follows from Lemma 1.2.

\section{REFERENCES}

1. J. R. Buddenhagen, Subsets of a countable set, Amer. Math. Monthly, 78 (1971), $536-537$.

2. P. L. Duren, Theory of $H^{p}$ Spaces, Academic Press, New York and London, 1970.

3. Otto Frostman, Potentiel d'équilibre et capacité des ensembles avec quelques applications à la theorie des fonctions, Meddel. Lunds Univ. Mat. Sem., 3 (1935), 1-118.

4. K. Hoffman, Banach Spaces of Analytic Functions, Prentice-Hall, Englewood Cliffs, New Jersey, 1962.

5. D. Sarason, Approximation of piecewise continuous functions by quotients of bounded analytic functions, Canad. J. Math., 24 (1972), 642-657. 
6. W. Seidel, On the distribution of values of bounded analytic functions, Trans. Amer. Math. Soc., 36 (1934), 201-226.

Received October 9, 1972, and in revised form April 23, 1973. This research was supported by National Science Foundation Grant GP-14255 and GU-3173.

UNIVERSITY OF CHICAGO'

AND

State University of New York at Albany

Present address: Universidad Nacional de Río IV ${ }^{\circ}$

Departamento de Matemáticas

Río IV ${ }^{\circ}$-Córdoba, Argentina 



\section{PACIFIC JOURNAL OF MATHEMATICS}

\section{EDITORS}

RICHARD ARENS (Managing Editor)

University of California

Los Angeles, California 90024

R. A. BeAumont

University of Washington

Seattle, Washington 98105
J. DugundJI*

Department of Mathematics

University of Southern California

Los Angeles, California 90007

D. Gilbarg and J. Milgram

Stanford University

Stanford, California 94305

\section{ASSOCIATE EDITORS}

E. F. BECKENBACH

B. H. NEUMANN

F. WOLF

K. YoSHIDA

\section{SUPPORTING INSTITUTIONS}

UNIVERSITY OF BRITISH COLUMBIA
CALIFORNIA INSTITUTE OF TECHNOLOGY
UNIVERSITY OF CALIFORNIA
MONTANA STATE UNIVERSITY
UNIVERSITY OF NEVADA
NEW MEXICO STATE UNIVERSITY
OREGON STATE UNIVERSITY
UNIVERSITY OF OREGON
OSAKA UNIVERSITY

UNIVERSITY OF BRITISH COLUMBIA CALIFORNIA INSTITUTE OF TECHNOLOGY UNIVERSITY OF CALIFORNIA MONTANA STATE UNIVERSITY NEW MEXICO STATE UNIVERSITY UNIVERSITY OF OREGON OSAKA UNIVERSITY
UNIVERSITY OF SOUTHERN CALIFORNIA STANFORD UNIVERSITY UNIVERSITY OF TOKYO UNIVERSITY OF UTAH WASHINGTON STATE UNIVERSITY UNIVERSITY OF WASHINGTON AMERICAN MATHEMATICAL SOCIETY NAVAL WEAPONS CENTER

* C. R. DePrima California Institute of Technology, Pasadena, CA 91109, will replace J. Dugundji until August 1974. 


\section{Pacific Journal of Mathematics}

\section{Vol. 51, No. $1 \quad$ November, 1974}

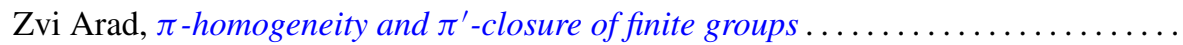

Ivan Baggs, A connected Hausdorff space which is not contained in a maximal

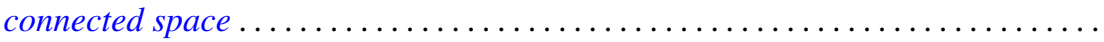

Eric Bedford, The Dirichlet problem for some overdetermined systems on the unit ball in $C^{n}$

R. H. Bing, Woodrow Wilson Bledsoe and R. Daniel Mauldin, Sets generated by

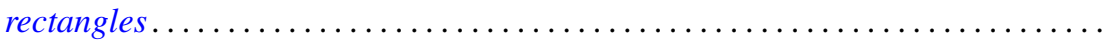

Carlo Cecchini and Alessandro Figà-Talamanca, Projections of uniqueness for

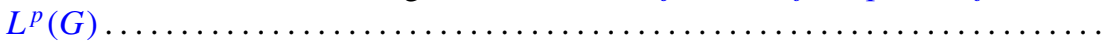

Gokulananda Das and Ram N. Mohapatra, The non absolute Nörlund summability of Fourier series .

Frank Rimi DeMeyer, On separable polynomials over a commutative ring ........ Richard Detmer, Sets which are tame in arcs in $E^{3} \ldots \ldots \ldots \ldots \ldots \ldots \ldots \ldots$

William Erb Dietrich, Ideals in convolution algebras on Abelian groups ..........

Bryce L. Elkins, A Galois theory for linear topological rings .................

William Alan Feldman, A characterization of the topology of compact convergence on $C(X)$.

Hillel Halkin Gershenson, A problem in compact Lie groups and framed cobordism

Samuel R. Gordon, Associators in simple algebras.

Marvin J. Greenberg, Strictly local solutions of Diophantine equations

Jon Craig Helton, Product integrals and inverses in normed rings . . . . . . . . . . . .

Domingo Antonio Herrero, Inner functions under uniform topology . . .

Jerry Alan Johnson, Lipschitz spaces .

Marvin Stanford Keener, Oscillatory solutions and multi-point boundary value

functions for certain nth-order linear ordinary differential equations.

John Cronan Kieffer, A simple proof of the Moy-Perez generalization of the

Shannon-McMillan theorem .......................

Joong Ho Kim, Power invariant rings

Gangaram S. Ladde and V. Lakshmikantham, On flow-invariant sets .

Roger T. Lewis, Oscillation and nonoscillation criteria for some self-adjoint even

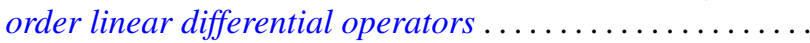

Jürg Thomas Marti, On the existence of support points of solid convex sets ..

John Rowlay Martin, Determining knot types from diagrams of knots . .

James Jerome Metzger, Local ideals in a topological algebra of entire functions

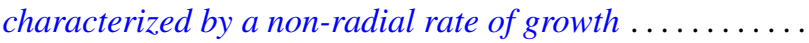

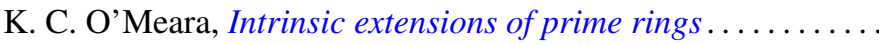

Stanley Poreda, A note on the continuity of best polynomial approximations ..

Robert John Sacker, Asymptotic approach to periodic orbits and local prolongations

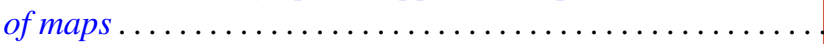

Eric Peter Smith, The Garabedian function of an arbitrary compact set . .

Arne Stray, Pointwise bounded approximation by functions satisfying a side condition

John St. Clair Werth, Jr., Maximal pure subgroups of torsion complete abelian

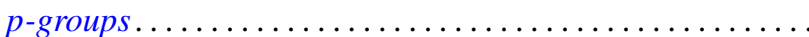

